\title{
STATISTICAL PROPERTIES OF A PARAMETRIC CHANNEL MODEL FOR MULTIPLE ANTENNA SYSTEMS
}

\author{
Salman Durrani ${ }^{\dagger}$, Marek E. Bialkowski* and Saba Latif ${ }^{\dagger}$ \\ $\dagger^{\dagger}$ Department of Engineering, The Australian National University, Canberra, Australia. \\ Email: salman.durrani@anu.edu.au \\ ${ }^{*}$ School of ITEE, The University of Queensland, Brisbane, Australia.
}

\begin{abstract}
Parametric channel models for Multiple Input Multiple Output (MIMO) systems have received much attention in recent years. This paper investigates the statistical properties of a parametric channel model for MIMO systems in an urban macrocell environment. We assess the performance of the proposed channel model (in terms of autocorrelation, cross-correlation, level crossing rate, average fade duration and spatial correlation at base and mobile station) by comparison with statistical properties of a reference MIMO channel model. We investigate the important problem of how many subpaths are sufficient to accurately model the statistical behaviour of the MIMO wireless channel. Comparison of the simulated and reference model results provides new insights into the statistical accuracy of the parametric channel modelling approach.
\end{abstract}

\section{INTRODUCTION}

Parametric channel models are an important class of channel models for Multiple Input Multiple Output (MIMO) systems [1]-[5]. Parametric channel models use important physical parameters such as phases, delays, doppler frequency, angle of departure (AOD), angle of arrival (AOA) and angle spread to provide a description of MIMO channel characteristics [6]. These parameters are described by prescribing underlying probability distribution functions without assuming an underlying geometry for scatterers (as is done in geometric models). When all the parameters are defined, they can be related using an analytical formulation. This can then be used to model different environments by choosing appropriate parameter values.

An example of a standardised model, based on the parametric approach, is the Spatial Channel Model (SCM) developed by Third Generation Partnership Project [5]. This is a detailed system level model which is applicable to urban micro-cell, urban macro-cell and suburban macro-cell fading environments. Although several similar implementations of parametric channel models can be found in the literature [1][4], less has been reported about the statistical properties of the resulting channel models. It is well known that the performance of MIMO systems is greatly influenced by the spatial and temporal properties of the underlying wireless propagation environment [7]. Thus it is important to investigate the statistical properties of parametric channel models.

In this work, we propose a parametric channel model for MIMO systems in an urban macro-cell environment (approximately $3 \mathrm{~km}$ base station separation as defined in [5]). We investigate the important problem of how many subpaths are sufficient to accurately model the statistical behaviour of the MIMO wireless channel. First, we summarise the statistical properties (autocorrelation, cross-correlation, level crossing rate, average fade duration and spatial correlation) of a reference MIMO channel model [8]-[10]. Next, we perform computer simulations based on proposed parameter values and compare the simulation results with those produced by closed-form expressions for the case of the reference MIMO channel model. This comparison provides new insights into the statistical accuracy and overall complexity of the parametric channel modelling approach.

This paper is organised as follows. The overall framework of the channel model is presented in Section II. The temporal and spatial properties of the reference MIMO channel model are described in Section III. The simulation results are discussed and compared with results offered by closed-form expressions in Section IV. Finally conclusions are drawn in Section V.

\section{PARAmetric Channel Model}

Without loss of generality, we consider a downlink system i.e. the Base Station (BS) is transmitting and Mobile Station (MS) is receiving. We assume a triangular BS with each side covering a $120^{\circ}$ sector. For simplicity, we assume that the BS and MS employ $N_{\mathrm{B}}$ element Uniform Linear Array (ULA) and an $N_{\mathrm{M}}$ element ULA with inter-element spacings $d_{\mathrm{B}}$ and $d_{\mathrm{M}}$, respectively. The transmitted signal from the BS reaches the MS via scattering from clusters. Following [4], [5], the received signal at the MS can be modelled as $L$ time delayed replicas of the transmitted signal with each path further consisting of $S$ subpaths. The paths (corresponding to a cluster) are considered to be resolvable while the subpaths (corresponding to scatterers in a cluster) are considered to be unresolvable. A simplified sketch of the model showing the angular parameters is given in Fig. 1.

\section{A. Channel Impulse Response}

The channel impulse response for the $l$ th path of the $k$ th user between the $n$th transmit and $m$ th receive antenna can be written as

$$
\begin{aligned}
h_{k, l}^{(m, n)}(t) & =\sqrt{\frac{\Omega_{k, l}}{S}}\left\{\sum_{s=1}^{S} \exp \left[j\left(\phi_{k, l}^{(s)}+2 \pi f_{D} t \cos \theta_{k, l, A O A}^{(s)}\right)\right]\right. \\
& \times \exp \left[-j \kappa d_{\mathrm{M}}(m-1) \sin \theta_{k, l, A O A}^{(s)}\right] \\
& \left.\times \exp \left[-j \kappa d_{\mathrm{B}}(n-1) \sin \theta_{k, l, A O D}^{(s)}\right]\right\} \delta\left(t-\tau_{k, l}\right)
\end{aligned}
$$




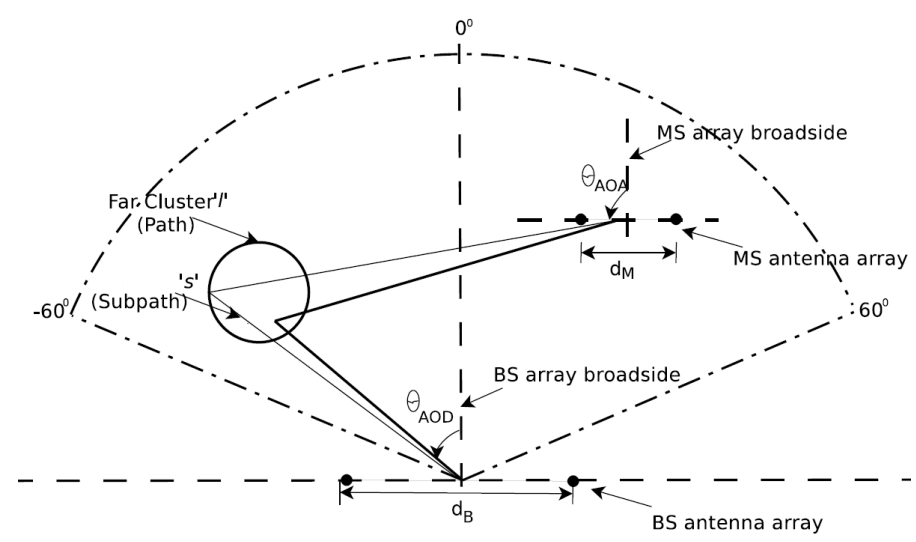

Fig. 1. Illustration of Parametric Channel Model angular parameters.

where $S$ is the number of subpaths ( $s$ is the subpath index), $L$ is the number of paths ( $l$ is the path index), $K$ is the number of users ( $k$ is the user index), $N_{\mathrm{B}}$ is the number of BS antennas ( $n$ is the BS antenna index), $N_{\mathrm{M}}$ is the number of MS antennas ( $m$ is the MS antenna index), $d_{\mathbf{M}}$ is the MS inter-element distance, $d_{\mathrm{B}}$ is the BS inter-element distance, $\kappa=2 \pi / \lambda$ is the wave number, $f_{D}$ is the maximum Doppler frequency, $\Omega_{k, l}$ is the mean path power for the lth path of the $k$ th user, $\tau_{k, l}$ is the signal time delay for the $l$ th path of the $k$ th user, $\phi_{k, l}^{(s)}$ is the random phase of the sth sub-path, $\theta_{k, l, A O A}^{(s)}=\theta_{k, A O A}+\vartheta_{k, l, A O A}^{(s)}$, where $\theta_{k, A O A}$ is the Mean AOA of the $k$ th user and $\vartheta_{k, l, A O A}^{(s)}$ is a zero mean uniform angular deviation, $\theta_{k, l, A O D}^{(s)}=\theta_{k, A O D}+\vartheta_{k, l, A O D}^{(s)}$, where $\theta_{k, A O D}$ is the Mean AOD of the $k$ th user and $\vartheta_{k, l, A O D}^{(s)}$ is a zero mean Gaussian angular deviation with standard deviation $\sigma_{A O D}$.

\section{B. Description of Channel Parameters}

The following assumptions are made with regard to the channel parameters:-

\section{Non Line-Of-Sight Multipath Propagation}

We consider multipath propagation with no Line-Of-Sight (LOS) component, which is appropriate for urban macrocell environments. The path powers and delays can be determined using standard power delay profiles [6]. If required, the existence of LOS component for urban microcell can also be taken into account using an approach described in [3].

\section{Angle of Departure and Angle of Arrival}

The mean Angle of Departure (AOD) from the BS is assumed to be uniformly distributed in $\left[-\frac{\pi}{3}, \frac{\pi}{3}\right]$ for a three-sector system. The mean AOA at the MS is dependent on local scatterers and the random orientation of MS and is assumed to be uniformly distributed in $[-\pi, \pi]$. The mean AOAs are measured from the respective array broadsides, as shown in Fig. 1.

\section{3. $P D F$ in $A O D$}

MIMO channel models assume either a Probability Density Function (PDF) for the distribution of received energy (Power Azimuth Spectrum (PAS)) or a PDF for the distribution of received signals (Azimuth Angular Spread (AAS)) respectively. Measurement have indicated that in typical urban environ- ments, the PAS is accurately described by a Laplacian distribution while the AAS is accurately described by a Gaussian distribution [11]. The median angular spread depends on the height of the BS antenna and typically varies in the range $5^{\circ}-20^{\circ}$, as reported in [11]. We adopt the AAS approach and assume that the azimuth signal distribution at the BS can be modelled by a Gaussian distribution.

4. $P D F$ in $A O A$

The PDF in the AOA describes the angular distribution of the subpaths arriving at the MS in azimuth. For simplicity, we assume Clark's isotropic scattering model [12], i.e. the azimuth signal distribution at the MS is modelled by a uniform PDF over $[-\pi, \pi]$. It must be noted that the general case of nonuniform angular distribution of the subpaths at the MS can be handled using the von Mises distribution [3].

\section{Channel Matrix}

Using (1), the general $N_{\mathrm{M}} \times N_{\mathrm{B}}$ channel matrix $\mathbf{H}_{k, l}$ can be written. For a $2 \times 2$ system, it is given by

$$
\mathbf{H}_{k, l}=\left[\begin{array}{ll}
h_{k, l}^{(1,1)} & h_{k, l}^{(1,2)} \\
h_{k, l}^{(2,1)} & h_{k, l}^{(2,2)}
\end{array}\right]
$$

where $h_{k, l}^{(m, n)}$ is the channel impulse response between MS antenna $m$ and BS antenna $n$.

\section{Properties of Reference Channel Model}

In this section, we describe the temporal and spatial properties of a reference MIMO channel model. The reference properties are summarized from [8]-[10]. The goal of the proposed parametric channel model is to reproduce these statistical properties as accurately as possible.

\section{A. Temporal Properties}

The channel coefficients in (1) are complex numbers and can be written as

$$
h_{k, l}^{m, n}(t)=\left(h_{I}\right)_{k, l}^{m, n}(t)+j\left(h_{Q}\right)_{k, l}^{m, n}(t)
$$

where $h_{I}(t)$ and $h_{Q}(t)$ represent the real and imaginary parts of the complex channel coefficient. Under isotropic scattering assumptions with number of subpaths $S \rightarrow \infty$, the temporal correlations are specified by the following functions [8]

$$
\begin{aligned}
R_{h_{I} h_{I}}(\tau) & =E\left[h_{I}(t) h_{I}(t+\tau)\right]=J_{0}\left(2 \pi f_{D} \tau\right) \\
R_{h_{Q} h_{Q}}(\tau) & =E\left[h_{Q}(t) h_{Q}(t+\tau)\right]=J_{0}\left(2 \pi f_{D} \tau\right) \\
R_{h_{I} h_{Q}}(\tau) & =E\left[h_{I}(t) h_{Q}(t+\tau)\right]=0 \\
R_{h h}(\tau) & =E\left[h(t) h^{*}(t+\tau)\right]=2 J_{0}\left(2 \pi f_{D} \tau\right) \\
R_{|h|^{2}|h|^{2}}(\tau) & =4+4 J_{0}^{2}\left(2 \pi f_{D} \tau\right)
\end{aligned}
$$

where $|h(t)|^{2}$ is the squared fading envelope, $E(\cdot)$ is the statistical average operator, $f_{D}$ is maximum Doppler frequency and $J_{0}(\cdot)$ is the zero-order Bessel function of the first kind.

Two other important second-order statistical properties are the Level Crossing Rate (LCR) and Average Fade Duration (AFD). LCR is defined as the rate at which the fading envelope crosses a specified threshold in the positive slope while AFD is the average duration of time that the fading envelope remains 
below a specified threshold. When $S \rightarrow \infty$, the LCR, $L_{|h|}$, and AFD, $T_{|h|}$, of fading envelope are given by [13]

$$
\begin{aligned}
L_{|h|} & =\sqrt{2 \pi} f_{D} \rho e^{-\rho^{2}} \\
T_{|h|} & =\frac{e^{\rho^{2}}-1}{\rho \sqrt{2 \pi} f_{D}}
\end{aligned}
$$

where $\rho$ is the specified envelope threshold.

\section{B. Spatial Properties}

The spatial envelope correlation coefficient $\rho_{s}$, between the $p$ th and $q$ th antenna elements for a ULA, is given by

$$
\rho_{s}(p, q)=\left|\Re\left\{R_{s}(p, q)\right\}+j \Im\left\{R_{s}(p, q)\right\}\right|^{2}
$$

where $R_{s}(p, q)$ denotes the complex spatial correlation between the $p$ th and $q$ th antenna elements for a ULA.

\section{Spatial Correlation at Mobile Station}

Assuming a uniform distribution for the AOA, the real and imaginary parts of signal correlation between the $p$ th and $q$ th MS antenna elements are given by [10]

$$
\begin{aligned}
\Re\left\{R_{s}(p, q)\right\} & =J_{0}\left(z_{p q}\right)+2 \sum_{v=1}^{\infty} J_{2 v}\left(z_{p q}\right) \cos \left(2 v \theta_{A O A}\right) \\
& \times \operatorname{sinc}(2 v \Delta) \\
\Im\left\{R_{s}(p, q)\right\} & =2 \sum_{v=0}^{\infty} J_{2 v+1}\left(z_{p q}\right) \sin \left[(2 v+1) \theta_{A O D}\right] \\
& \times \operatorname{sinc}[(2 v+1) \Delta]
\end{aligned}
$$

where $\theta_{A O A}$ is the mean AOA, $\Delta$ is the scattering angle (corresponding to MS angle spread $\sigma_{A O A}=\frac{\Delta}{\sqrt{3}}$ ), $z_{p q}=$ $2 \pi|p-q| d_{\mathrm{M}} / \lambda, d_{\mathrm{M}}$ is the MS inter-element distance and $\operatorname{sinc}(x)=\frac{\sin (x)}{x}$ is the $\operatorname{sinc}(\cdot)$ function.

2. Spatial Correlation at Base Station

Assuming a Gaussian distribution for the AOD, the real and imaginary parts of the signal correlation between the $p$ th and $q$ th BS antenna elements are given by [9]

$$
\begin{aligned}
\Re\left\{R_{s}(p, q)\right\} & =J_{0}\left(z_{p q}\right)+2 C_{g} \sum_{v=1}^{\infty} J_{2 v}\left(z_{p q}\right) \cos \left(2 v \theta_{A O D}\right) \\
& \times \exp \left(-2 v^{2} \sigma_{A O D}^{2}\right) \Re\left\{\operatorname{erf}\left(\frac{\pi+j 2 v \sigma_{A O D}^{2}}{\sqrt{2} \sigma_{A O D}}\right)\right\} \\
\Im\left\{R_{s}(p, q)\right\} & =2 C_{g} \sum_{v=0}^{\infty} J_{2 v+1}\left(z_{p q}\right) \sin \left[(2 v+1) \theta_{A O D}\right] \\
& \times \exp \left[\frac{-(2 v+1)^{2} \sigma_{A O D}^{2}}{2}\right] \\
& \times \Re\left\{\operatorname{erf}\left(\frac{\pi+j(2 v+1) \sigma_{A O D}^{2}}{\sqrt{2} \sigma_{A O D}}\right)\right\} \quad(13 \mathrm{~b})
\end{aligned}
$$

(13a) fading enved

(13a) fading envelope is significant because it is proportional to

where $\theta_{A O D}$ is the mean AOD, $\sigma_{A O D}$ is the BS angle spread, $z_{p q}=2 \pi|p-q| d_{\mathrm{B}} / \lambda, d_{\mathrm{B}}$ is the BS inter-element distance, $\lambda$ is the wavelength, $J_{n}(x)$ is the $n$th order Bessel function of the first kind, $C_{g}=\frac{1}{\operatorname{erf}\left(\pi / \sqrt{2} \sigma_{A O D}\right)}$ is a constant and $\operatorname{erf}(a+j b)$ is the complex input error function [14]. instantaneously received signal power. Fig. 3 shows the crosscorrelation of real and imaginary parts of $h^{(2,2)}(t)$. The normalised empirical correlations from the simulations were calculated using [16]

$$
R_{a b}[n]=\frac{\sum_{k=1}^{N-n} a^{*}[k] b[n+k]}{\sqrt{\sum_{k=1}^{N-n}|a[k]|^{2} \sum_{k=1}^{N-n}|b[k]|^{2}}}
$$

The expressions in (12) and (13) can be substituted into (11) to obtain the spatial envelope correlation at the MS and the BS respectively.

\section{RESUlts}

The simulation results in this section are obtained by $2 \times 2$ MIMO system is considered and $T=20000$ channel coefficients are generated with 8 samples/wavelength and mobile velocity $v=60 \mathrm{~km} / \mathrm{hr}\left(f_{c}=2 \mathrm{GHz}, f_{D}=111.11\right.$ $\mathrm{Hz})$. This corresponds to a normalised distance travelled by the MS of $d / \lambda=1250$, which is sufficient to represent the statistics accurately [15]. The number of subpath best tradeoff between statistical model accuracy and model complexity (in comparison, $S=20$ is adopted in the SCM specifications [5]). The parameters values for urban macrocell, used in the simulations, are summarized in Table I.

\section{TABLE I}

\begin{tabular}{l|l|l}
\hline Aspect & Parameter & Value or Description \\
\hline \multirow{4}{*}{ General } & Carrier frequency & $f_{c}=2 \mathrm{GHz}$ \\
& Number of channel samples & $T=20000$ \\
& Samples/wavelength & 8 \\
\hline \multirow{5}{*}{ Temporal } & MS velocity & $v=60 \mathrm{~km} / \mathrm{hr}$ \\
& Channel environment & Rayleigh fading \\
& Number of paths & $L=1$ \\
& Number of subpaths & $S=25$ \\
\hline \multirow{5}{*}{ Antennas } & Antenna geometry & $\mathrm{ULA}$ \\
& Number of BS transmit antennas & $N_{\mathrm{B}}=2$ \\
& Number of MS receive antennas & $N_{\mathrm{M}}=2$ \\
& BS Inter-element distance & $d_{\mathrm{B}}=5 \lambda$ \\
& MS Inter-element distance & $d_{\mathrm{M}}=0.5 \lambda$ \\
\hline \multirow{5}{*}{ Spatial } & Angle of Arrival & $\theta_{A O A}=60^{\circ}$ \\
& pdf in Angle of Arrival & Uniform $(\Delta=\pi)$ \\
& Angle of Departure & $\theta_{A O D}=0^{\circ}$ \\
& pdf in Angle of Departure & Gaussian \\
& BS Angle spread & $\sigma_{A O D}=5^{\circ}, 10^{\circ}, 20^{\circ}$ \\
\hline \multicolumn{2}{|l}{}
\end{tabular}

First, we investigate the temporal properties of the proposed channel model using $h^{(2,2)}(t)$. Fig. 2 shows the autocorrelation of the real part of $h^{(2,2)}(t)$ and the autocorrelation of the squared fading envelope $\left|h^{(2,2)}(t)\right|^{2}$ respectively. The squared where $a[n]$ and $b[n]$ are the $n$th samples of amplitude sequences $(b[n]=a[n]$ for autocorrelations), $N$ is the total number of samples and $(\cdot)^{*}$ denotes complex conjugate.

Figs. 2 and 3 show that the simulation results match perfectly with the reference model results up to about $3 f_{D} \tau$. In

\section{A. Temporal Correlation Characteristics}




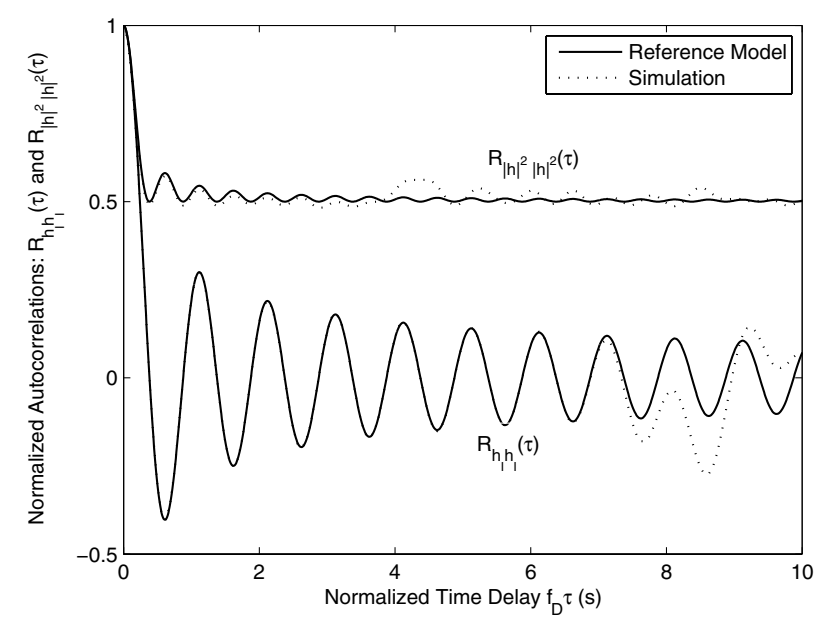

Fig. 2. Autocorrelations of the real part of $h^{(2,2)}(t)$ and squared fading envelope $\left|h^{(2,2)}(t)\right|^{2}$ respectively.

most communication systems, short time delays e.g. $f_{D} \tau \leq 2$ are of more interest [17]. Hence the proposed channel model is excellent in terms of the temporal correlation properties. The temporal behaviour of the remaining channel sequences in (2) is similar and therefore not shown here.

\section{B. Level Crossing Rate and Average Fade Duration}

Fig. 4 shows the plot of the normalised LCR and AFD verses normalised threshold $\rho(\mathrm{dB})$ using a double axis. The reference model results from (9) and (10) are also included in the figure. It can be seen that the reference model and simulated results are in excellent agreement between the range $\rho=-15 \mathrm{~dB}$ and $\rho=10 \mathrm{~dB}$. For low threshold values $\rho \leq-20 \mathrm{~dB}$, the simulated results deviate slightly from the reference results.

From Figs. 2, 3 and 4, it can be concluded that the proposed model can accurately characterise the first and second order temporal statistics of the channel.

\section{Spatial Correlation at MS}

The plot of the spatial envelope correlation coefficient $\rho_{s}(1,2)$ at the MS for mean angle of arrival $\theta_{A O A}=60^{\circ}$ is shown in Fig. 5. The normalised empirical spatial envelope correlation coefficient from simulations is calculated using

$$
\rho_{s}=\left|\frac{E\left[c d^{*}\right]}{\sqrt{E\left[|c|^{2}\right] E\left[|d|^{2}\right]}}\right|^{2}
$$

where $c=h^{(1,1)}$ and $d=h^{(2,1)}$.

The figure shows that there is an excellent agreement between the simulated and reference model values for $0 \leq$ $d_{\mathrm{M}} / \lambda \leq 3.5$. The simulated results deviate very slightly from the reference model results only for higher values of normalised inter-element distance (which are unlikely to be utilized at the MS in a practical scenario). The results for other AOAs are similar and not shown here due to limited available space.

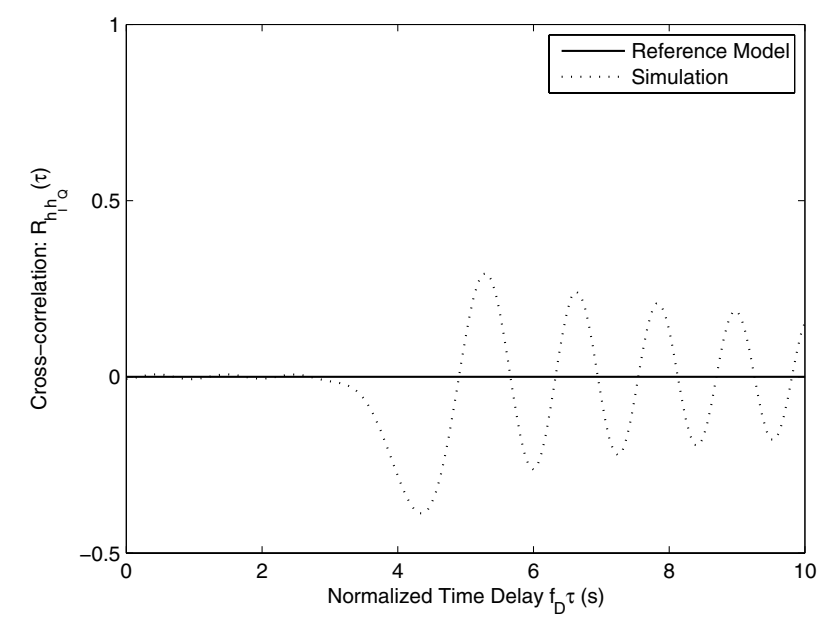

Fig. 3. Cross-correlation of the real and imaginary parts of $h^{(2,2)}(t)$.

\section{Spatial Correlation at $B S$}

Figs. 6 and 7 show the plots of spatial envelope correlation coefficient $\rho_{s}(1,2)$ at the BS for mean angle of departure $\theta_{A O D}=0^{\circ}$ (broadside) and $\theta_{A O D}=60^{\circ}$ (sector edge) and angle spreads $\sigma_{A O D}=5^{\circ}, 10^{\circ}, 20^{\circ}$ respectively. This range of BS angle spread values has been reported by measurements in typical outdoor environments [11]. The normalised empirical spatial envelope correlation coefficient from simulations is calculated using (15) with $c=h^{(1,1)}$ and $d=h^{(1,2)}$.

The figures show that for $0 \leq d_{\mathrm{B}} / \lambda \leq 3$ there is good match between the simulation and reference model values. For $d_{\mathrm{B}} / \lambda \geq 3$ and low values of correlation $\left(\rho_{s}<0.2\right)$ there is some mismatch. However, this mismatch does not adversely affect the results when the proposed model is used in system level simulations for multiple antenna systems [18].

\section{CONCLUSions}

In this paper, we have analysed the statistical properties of a parametric channel model for MIMO systems in an urban macrocell environment. The performance assessment is based on comparison with properties of a reference MIMO channel model. It has been shown that the proposed channel model can accurately represent the temporal correlations for time delays $0 \leq f_{D} \tau \leq 3$. It can accurately represent the spatial correlation at BS and MS for inter-element spacings $0 \leq d / \lambda \leq 3$. For larger inter-element spacings at the BS, there is slight mismatch for small correlation values $(<0.2)$. The obtained results show that the value of $S=25$ subpaths is sufficient to capture the important statistical behaviour of the MIMO wireless channel.

\section{REFERENCES}

[1] A. F. Molisch, "A generic model for MIMO wireless propagation channels in macro- and microcells," IEEE Trans. Signal Processing, vol. 52, no. 1, pp. 61-71, Jan. 2004.

[2] H. Xu, D. Chizhik, H. Huang, and R. Valenzuela, "A generalized spacetime multiple-input multiple-output (MIMO) channel model," IEEE Trans. Wireless Commun., vol. 3, no. 3, pp. 966-974, May 2004. 

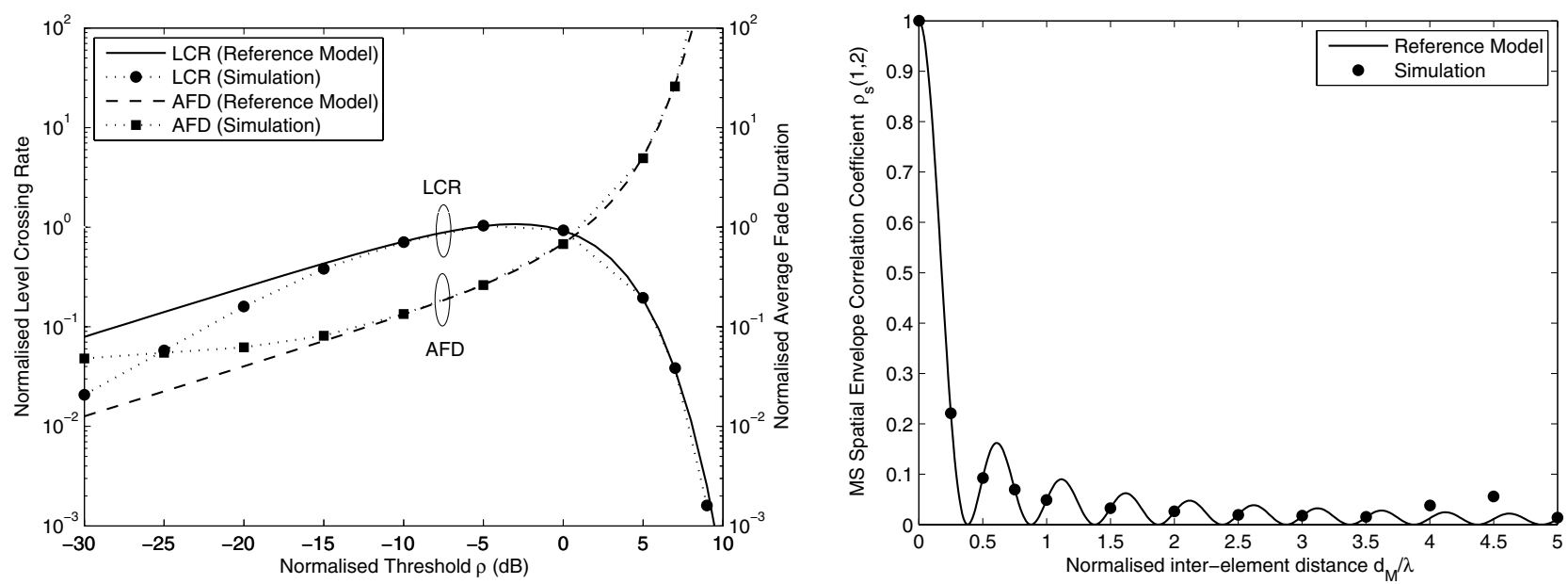

Fig. 4. Level crossing rate (LCR) and Average fade duration (AFD) of fading Fig. 5. Spatial envelope correlation coefficient at MS for mean AOA $\theta=60^{\circ}$. envelope $\left|h^{(2,2)}(t)\right|$.

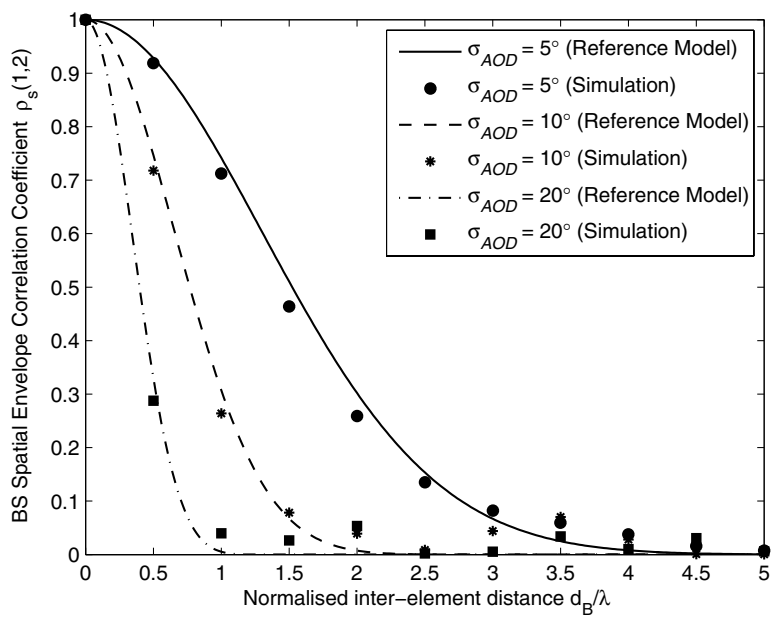

Fig. 6. Spatial envelope correlation coefficient at BS for mean AOD $\theta=0^{\circ}$ (broadside) and angle spread $\sigma_{A O D}=0^{\circ}, 10^{\circ}, 20^{\circ}$.

[3] A. Abdi, J. A. Barger, and M. Kaveh, "A parametric model for the distribution of the angle of arrival and the associated correlation function and power spectrum at the mobile station," IEEE Trans. Veh. Technol., vol. 51, no. 3, pp. 425-433, May 2002.

[4] S. Durrani and M. E. Bialkowski, "A parametric channel model for smart antennas incorporating mobile station mobility," in Proc. IEEE VTC, vol. 6, Melbourne, Australia, May 7-10, 2006, pp. 2803-2807.

[5] Third Generation Partnership Project Two (3GPP2), "Spatial Channel Model for Multiple Input Multiple Output (MIMO) simulations (3GPP TR 25.996)," Sept. 2003.

[6] K. Yu and B. Ottersten, "Models for MIMO propagation channels: A review," Wireless Communications and Mobile Communications, vol. 2, no. 7, pp. 653-666, Nov. 2002.

[7] P. J. Smith and M. Shafi, "The impact of complexity in MIMO channel models," in Proc. IEEE ICC, Paris, France, June 2004, pp. 2924-2928.

[8] C. S. Patel, G. L. Stuber, and T. G. Pratt, "Comparative analysis of statistical models for the simulation of rayleigh faded cellular channels," IEEE Trans. Commun., vol. 53, no. 6, pp. 1017-1026, June 2005.

[9] J. Luo, J. Zeidler, and S. McLaughlin, "Performance ananlysis of compact antenna arrays with MRC in correlated nakagami fading channels," IEEE Trans. Veh. Technol., vol. 50, no. 1, pp. 267-277, Jan. 2001.

[10] J. Salz and J. H. Winters, "Effect of fading correlation on adaptive arrays in digital mobile radio," IEEE Trans. Veh. Technol., vol. 43, no. 4, pp. 1049-1057, Nov. 1994.

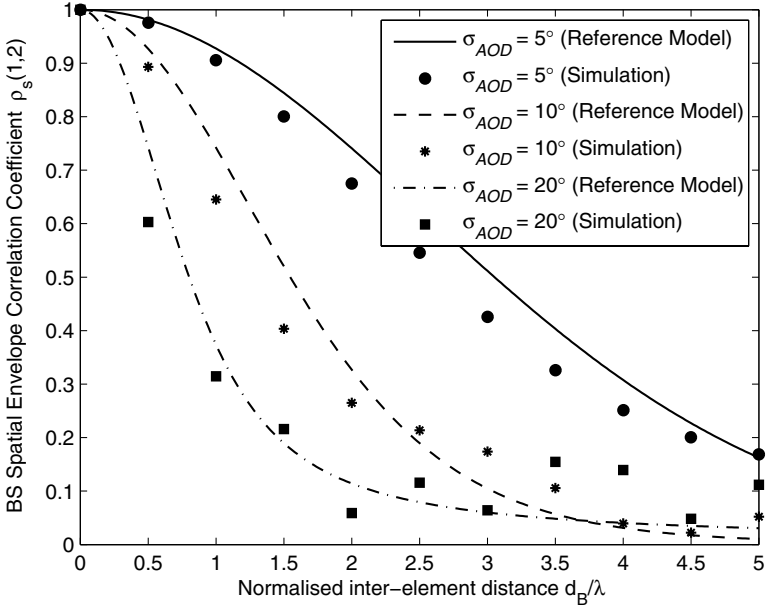

Fig. 7. Spatial envelope correlation coefficient at BS for mean AOD $\theta=60^{\circ}$ (sector edge) and angle spread $\sigma_{A O D}=0^{\circ}, 10^{\circ}, 20^{\circ}$.

[11] K. I. Pedersen and P. E. Mogensen, "A stochastic model of the temporal and azimuth dispersion seen at the base station in outdoor propagation environments," IEEE Trans. Veh. Technol., vol. 49, no. 2, pp. 437-447, Mar. 2000.

[12] R. H. Clark, "A statistical theory of mobile radio reception," Bell Labs Syst. Tech. J., vol. 47, pp. 957-1000, July-Aug. 1968.

[13] Y. R. Zheng and C. Xiao, "Simulation models with correct statistical properties for Rayleigh fading channels," IEEE Trans. Commun., vol. 51, no. 6, pp. 920-928, June 2003

[14] M. Abramowitz and I. A. Stegun, Handbook of Mathematical Functions: With Formulas, Graphs, and Mathematical Tables. Dover, 1965.

[15] G. S. Prabhu and M. Shankar, "Simulation of flat fading using matlab for classroom instruction," IEEE Transactions on Education, vol. 45 , no. 1, pp. 19-25, Feb. 2002.

[16] N. C. Beaulieu and C. Cheng, "Efficient Nakagami- $m$ fading channel simulation," IEEE Trans. Veh. Technol., vol. 54, no. 2, pp. 413-424, Mar. 2005.

[17] C.-X. Wang, M. Patzold, and D. Yuan, "Accurate and efficient simulation of multiple uncorrelated Rayleigh fading waveforms," IEEE Trans. Wireless Commun., vol. 6, no. 3, pp. 833-839, Mar. 2007.

[18] S. Durrani and M. E. Bialkowski, "Analysis of the error performance of adaptive array antennas for CDMA with noncoherent $M$-ary orthogonal modulation in Nakagami fading," IEEE Commun. Lett., vol. 9, no. 2, pp. 148-150, Feb. 2005. 